\title{
Study of DNA/RNA Aggregation Linked to Cadmium Oxide (CdO) Nanoparticles by Aryl Mercaptanes with Various Chain Length
}

\author{
Alireza Heidari ${ }^{1,2,3,4}$ \\ ${ }^{1}$ Faculty of Chemistry, California South University, 14731 Comet St. Irvine, CA 92604, USA \\ e-mail: Scholar.Researcher.Scientist@gmail.com, Alireza.Heidari@calsu.us,Central@aisi-usa.org \\ ${ }^{2}$ BioSpectroscopy Core Research Laboratory, California South University, 14731 Comet St. Irvine, \\ CA 92604, USA \\ ${ }^{3}$ Cancer Research Institute (CRI), California South University, 14731 Comet St. Irvine, CA 92604, USA \\ ${ }^{4}$ American International Standards Institute, Irvine, CA 3800, USA
}

\begin{abstract}
CdO nanoparticles show a strong peak of Plasmon absorption in ultraviolet-visible zone. A strong interaction exists between the surface of $\mathrm{CdO}$ nanoparticles and aryl mercaptan compounds. Aryl mercaptan compounds cause to aggregation of $\mathrm{CdO}$ nanoparticles linked to DNA/RNA and hence, lead to widening of peak Plasmon of CdO nanoparticles surface at $550(\mathrm{~nm})$ and emerging a new peak at higher wavelength. In the current project, this optical characteristic of $\mathrm{CdO}$ nanoparticles is used to time investigate of interaction between different aryl mercaptanes and $\mathrm{CdO}$ nanoparticles. The results were shown that aryl mercaptan compounds with shorter chain length interact faster with $\mathrm{CdO}$ nanoparticles. Therefore, a simple and fast method for identification of aryl mercaptanes with various chain length using red shift in surficial Plasmon absorption is presented.
\end{abstract}

Received: February 5, 2022; Accepted: March 6, 2022

Keywords and phrases: aryl mercaptans; peak Plasmon absorption; aggregation; CdO nanoparticles; DNA/RNA.

Copyright (C) 2022 Alireza Heidari. This is an open access article distributed under the Creative Commons Attribution License (http://creativecommons.org/licenses/by/4.0/), which permits unrestricted use, distribution, and reproduction in any medium, provided the original work is properly cited. 


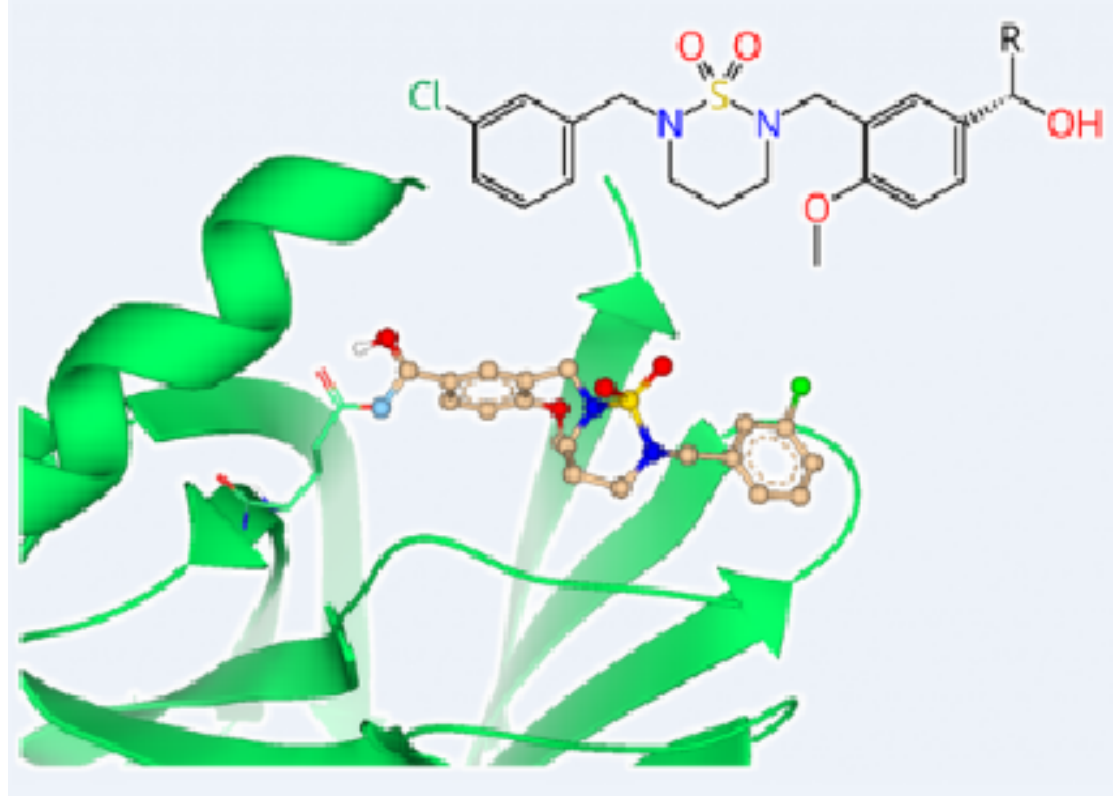

Schematic of Cadmium Oxide (CdO) Nanoparticles Aggregation Linked to DNA/RNA by Aryl Mercaptanes with Various Chain Length.

\section{Introduction}

Investigations about $\mathrm{CdO}$ nanoparticles are widely developed due to their considerable optical characteristics and potential application in optical devices, sensors and optical circuits specially in diagnostic and treating medical sciences [1-11]. CdO nanoparticles show a strong absorption peak in ultraviolet-visible zone when interact with light. The maximum position of spectrum depends on size, form, inter-particle space and de-electric environment of nanoparticles [12-21].

There is a high affinity between aryl groups and $\mathrm{CdO}$ nanoparticles which leads to aggregation of $\mathrm{CdO}$ nanoparticles linked to DNA/RNA. As a result of this aggregation, the Plasmon absorption peak of $\mathrm{CdO}$ nanoparticles widen at $550(\mathrm{~nm})$ and a new peak emerges at higher wavelength. Numerous researches have been performed about $\mathrm{CdO}$ nanoparticles aggregation linked to DNA/RNA and application of this characteristic of $\mathrm{CdO}$ nanoparticles for identification of target analytes and producing sensors [22-39]. In a research, chemical absorption of aryl mercaptans on $\mathrm{CdO}$ colloid at the presence of sodium hydroxide was investigated; the results were shown that the largeness of these changes depends on $\mathrm{pH}$, chain length and the end of aryl mercaptans chain [40-56]. At 
another research, the effective factors on controlling the optical characteristics of $\mathrm{CdO}$ nanoparticles aggregation linked to DNA/RNA including oligonucleotides linked with various lengths (72-24 pairs) were studied. This test was shown that optical characteristics of DNA/RNA aggregation linked to $\mathrm{CdO}$ nanoparticles are controlled with size of aggregation and ignoring the chain length of oligonucleotides which is important for colorimetric identification based on nanoparticle, it was shown that optical effects are more dependent to size of aggregation which in turn, it is under kinetic control [57-69]. The rate of band change of surface Plasmon is conversely related to the length of DNA/RNA connections so that 24 chains systems (shortest) have shown the highest change in rate [70-81].

Mercaptanes are important compounds in chemical synthesizes, environment, gas and petrochemical industries and biology [82-99]. In the current research, optical characteristic of $\mathrm{CdO}$ nanoparticles is used to time identification of aryl mercaptanes with various chain length. In previously used methods for identification of mercaptanes in petrochemical and oil industry, only total mercaptan could be identified; however, the current method can identify mercaptanes with various chain lengths which is very important for making sensors of these compounds [100-156].

\section{Materials and Experimental Methodology and Techniques}

\subsection{Preparing $\mathrm{CdO}$ nanoparticles and description of $\mathrm{CdO}$ nanoparticles aggregation linked to DNA/RNA}

All glass wears used in this test was washed with a solution of $\mathrm{HCl}: \mathrm{HNO}_{3}$ with concentration ratio of 3:1 and then, with deionized water and acetone and afterwards, dried in air. In this project, Terkovic method was used for synthesizing the $\mathrm{CdO}$ nanoparticles. A 0.05 (gr) of hydrogen tetra coloro-urate $\left(\mathrm{HCdCl}_{4}, 3 \mathrm{H}_{2} \mathrm{O}\right)$ was solved in $100(\mathrm{ml})$ of water and then, was indirectly heated under $150^{\circ} \mathrm{C}$ temperature and stirring rate of $500(\mathrm{rpm})$ in a balloon connected to a cooler. When $\mathrm{CdO}$ solution was boiled, 2.5 (ml) solution of sodium citrate of $0.05(\mathrm{M})$ was added and the colloidal solution of $\mathrm{CdO}$ was gradually produced with reduction of $\mathrm{CdO}$ ions (III). The color of initial solution was mellow yellow. The color of this solution was gradually changed to blue, violet and dark red. At the end of test, the color was dark red. The size of produced nanoparticles was 25 $(\mathrm{nm})$. The size of $\mathrm{CdO}$ nanoparticles was determined by DLS. In order to time investigate the interaction of $\mathrm{CdO}$ nanoparticles, aryl mercaptanes with various lengths were added to $\mathrm{CdO}$ nanoparticles at room temperature. 


\section{Results and Discussion}

The absorption spectrum of $\mathrm{CdO}$ nanoparticles was recorded in various times with aryl mercaptans with various chain lengths as shown in Figures 1, 2 and 3. As can be seen in these figures, peak is decreased at $573(\mathrm{~nm})$ and a new peak is emerged at higher wavelength which gradually increased with time and after reaching to the maximum, the absorption decreases which is due to complete aggregation linked to DNA/RNA and instability of the produced $\mathrm{CdO}$ nanoparticles.

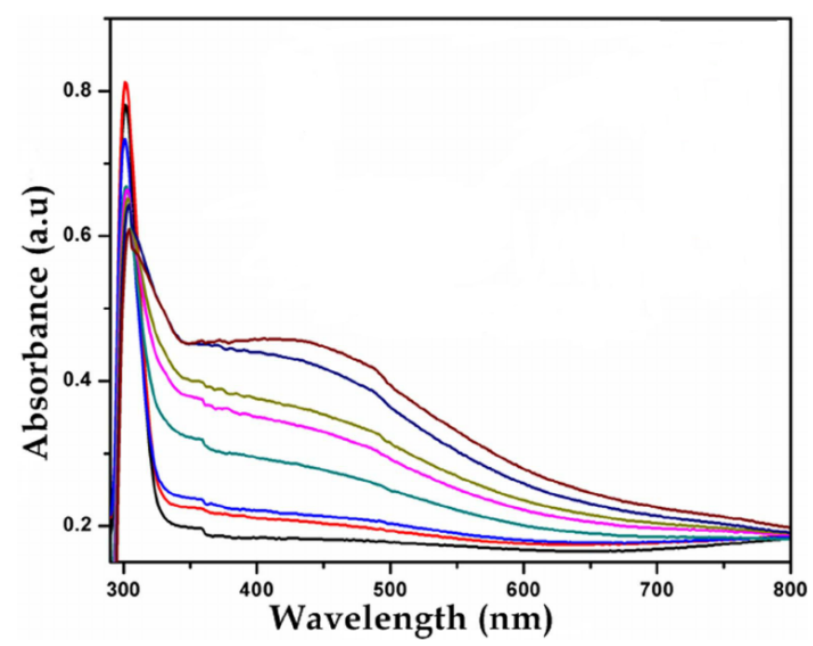

(a)

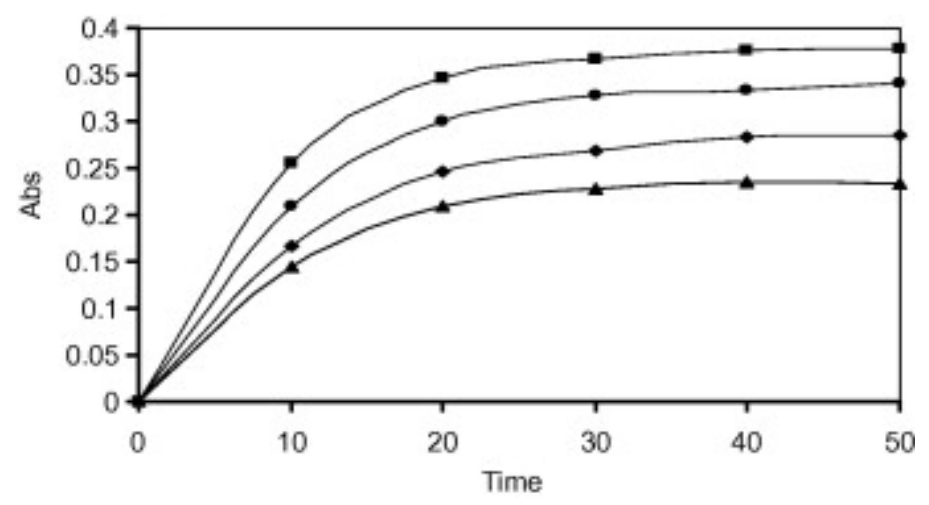

(b)

Figure 1. (a) Absorption spectrum of $\mathrm{CdO}$ nanoparticles-phenyl mercaptan during 01200 (s). (b) Absorption curve against time for $\mathrm{CdO}$ nanoparticles-phenyl mercaptan at maximum wavelength. 


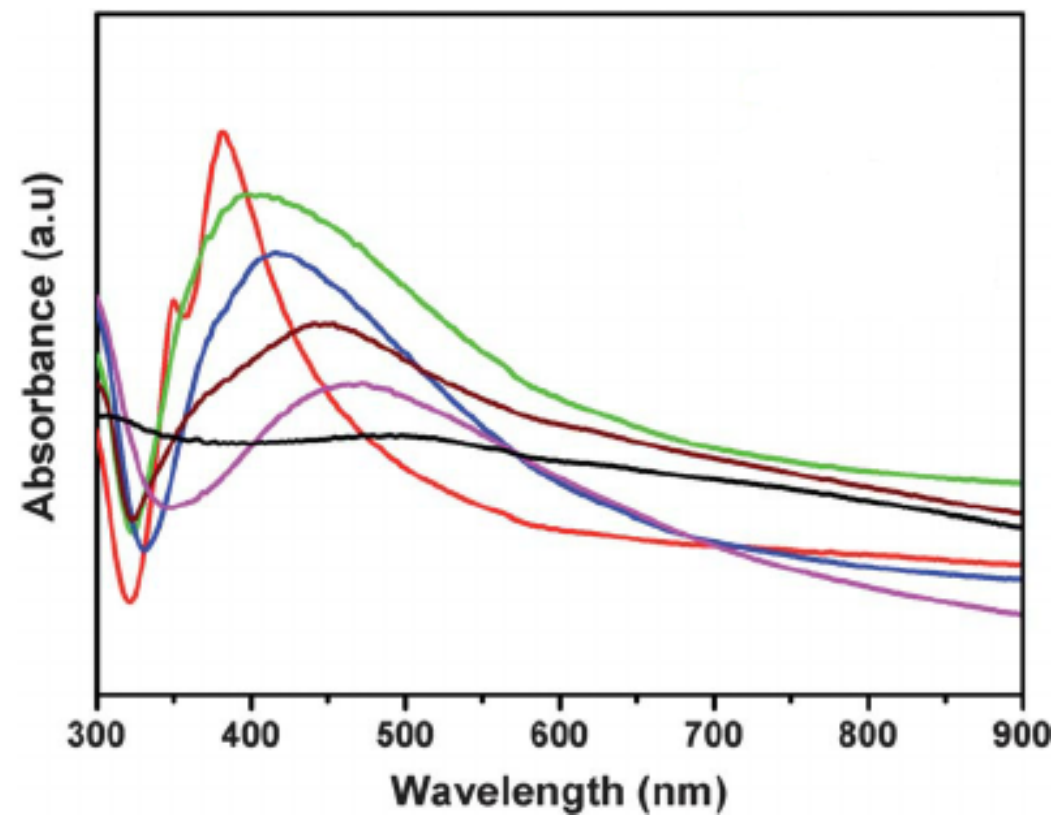

(a)

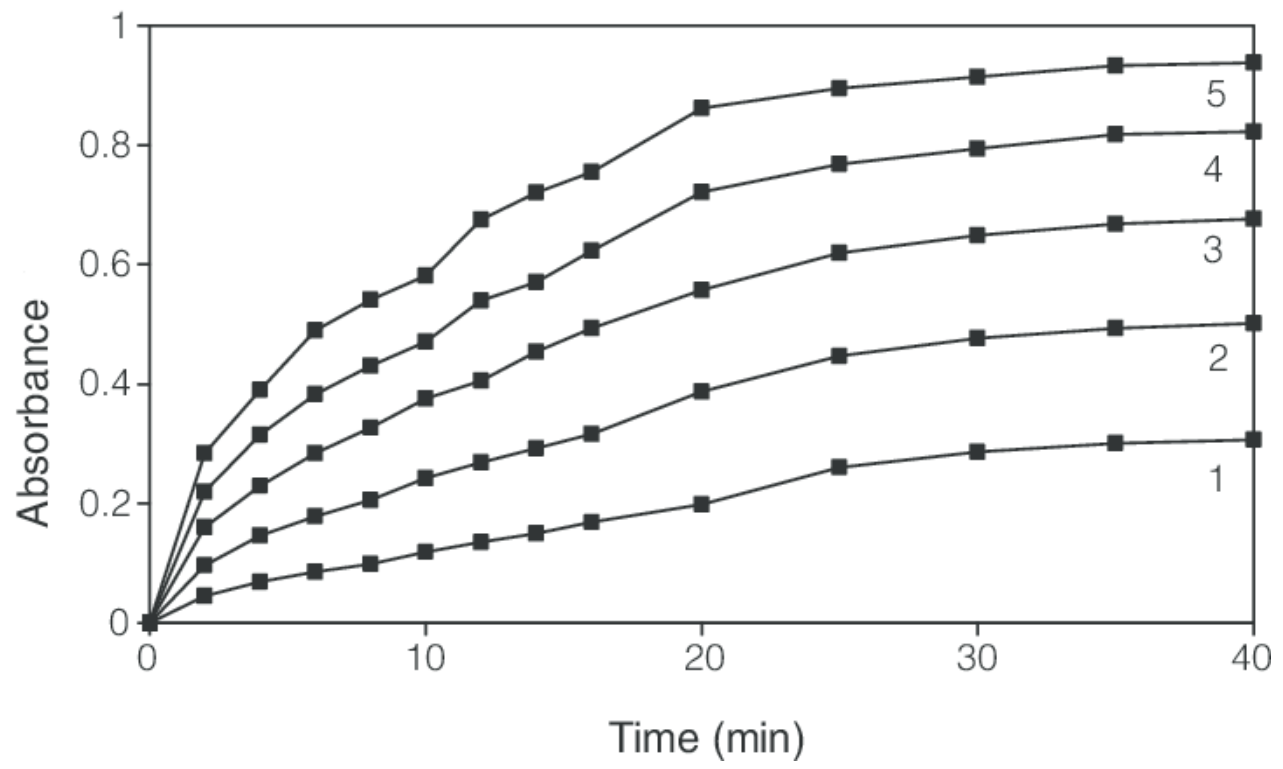

(b)

Figure 2. (a) Absorption spectrum of $\mathrm{CdO}$ nanoparticles-benzyl mercaptan during 01200 (s). (b) Absorption curve against time for $\mathrm{CdO}$ nanoparticles-benzyl mercaptan at maximum wavelength. 


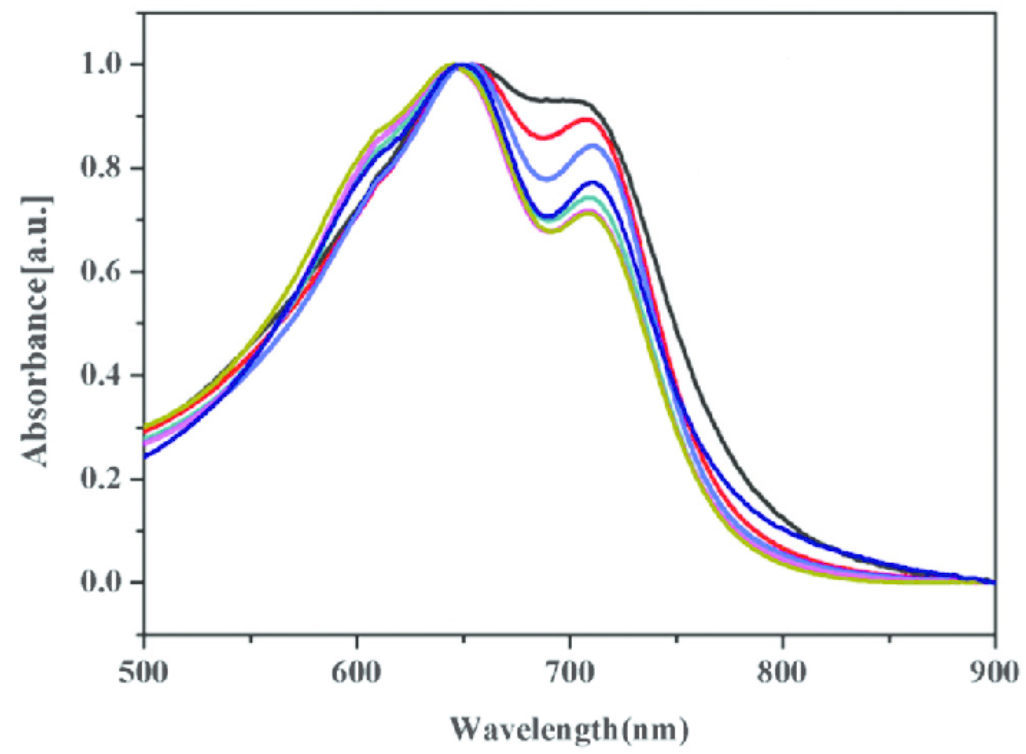

(a)

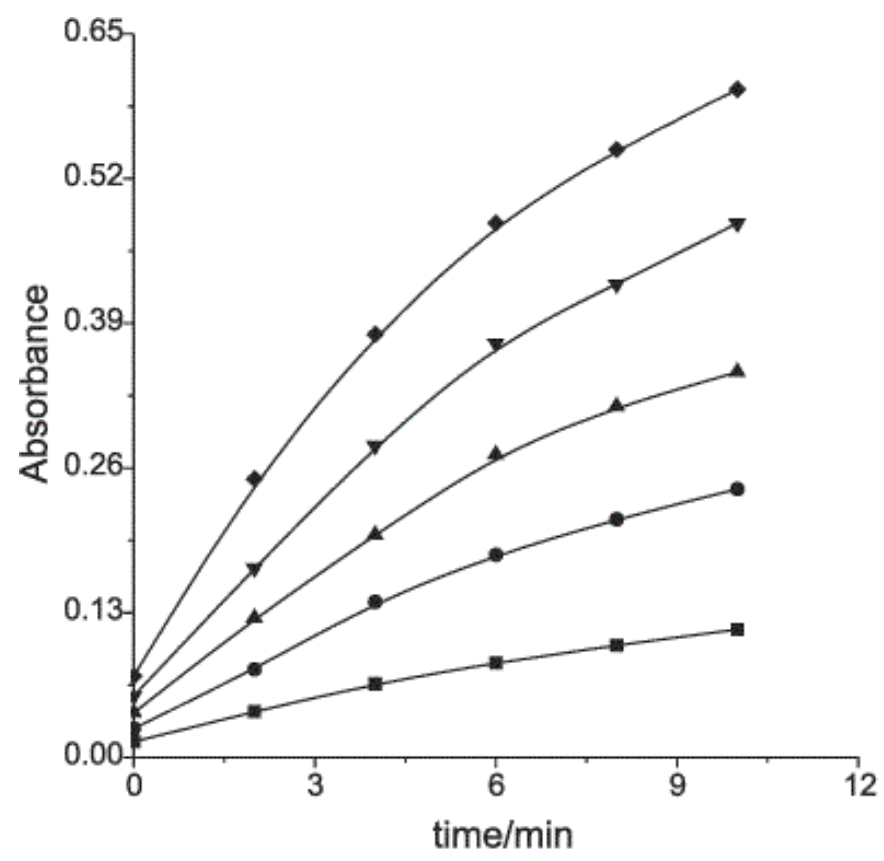

(b)

Figure 3. (a) Absorption spectrum of $\mathrm{CdO}$ nanoparticles-propyl mercaptan during 0-1200 (s). (b) Absorption curve against time for $\mathrm{CdO}$ nanoparticles-propyl mercaptan at maximum wavelength. 
The results show that aryl mercaptanes with shorter chain length lead to faster aggregation of $\mathrm{CdO}$ nanoparticles linked to DNA/RNA than ones with longer chain length. In other words, at shorter time, $\mathrm{CdO}$ nanoparticles is aggregated with aryl mercaptanes with shorter chain length at higher wavelength compared to absorption spectrum of $\mathrm{CdO}$ nanoparticles aggregated with aryl chains with longer chain length. As can be seen in Figure 4, during 90 (s), phenyl mercaptan is emerged at higher wavelength $(812.49 \mathrm{~nm})$ than phenyl $(777.91 \mathrm{~nm})$ and pentylmercaptanes (a wide peak between 500$760 \mathrm{~nm}$ ) and hence, aryl chains with various chain length can be identified through controlling the aggregation time.

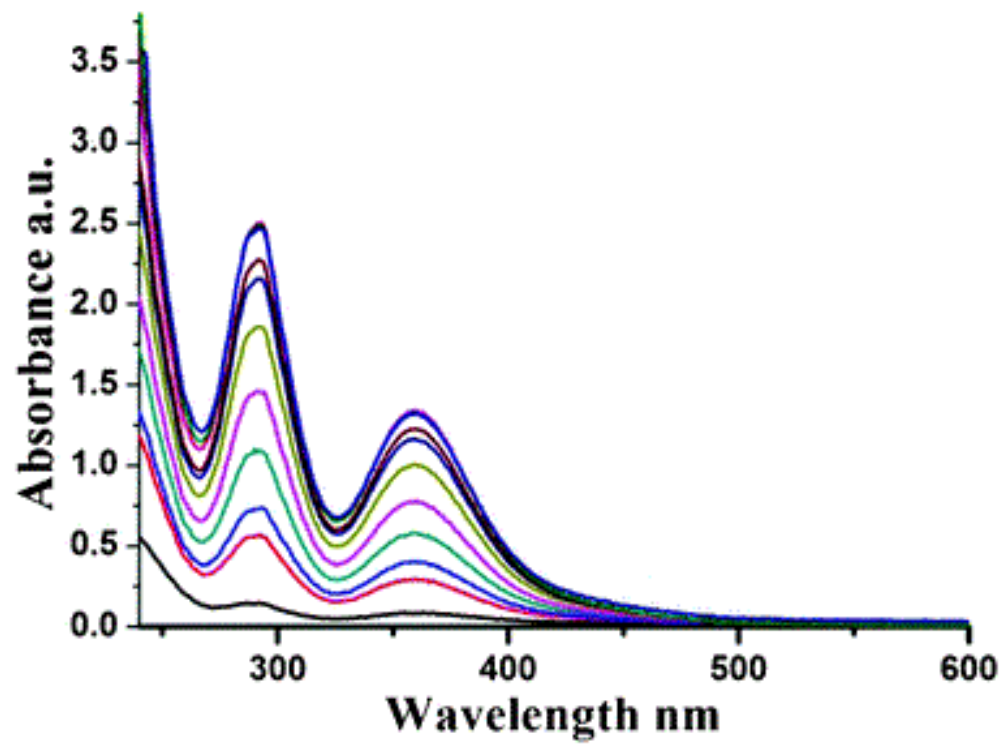

Figure 4. Absorption spectrum of $\mathrm{CdO}$ nanoparticles with various aryl mercaptanes during 90 (s) (concentration of CdNPs is equal to $250 \mathrm{ppm}$ and $2.5 \mathrm{ml}$ used, phenyl mercaptan $60 \mathrm{nmol}$, phenyl mercaptan $45.5 \mathrm{nmol}$ and phenyl mercaptan $55.5 \mathrm{nmol}$ ).

The optical difference for aggregation of aryl mercaptanes connected to chains with various lengths to $\mathrm{CdO}$ nanoparticles can be attributed to size of aggregation linked to DNA/RNA and inter-particle distance.

\section{Conclusion}

In the current study, optical characteristics of peal Plasmon of absorption of $\mathrm{CdO}$ nanoparticles was used to identify mercaptanes with various chain length and through time controlling, they were identified successfully. It was observed that the second peak 
at wavelength between $500-760(\mathrm{~nm})$ induced by interaction of mercaptanes with $\mathrm{CdO}$ nanoparticles in aryl mercaptanes with shorter chain length at shorter time duration observe at higher wavelength than aryl mercaptanes with longer wavelength.

\section{Acknowledgement}

This study was supported by the Cancer Research Institute (CRI) Project of Scientific Instrument and Equipment Development, the National Natural Science Foundation of the United Sates, the International Joint BioSpectroscopy Core Research Laboratory Program supported by the California South University (CSU), and the Key project supported by the American International Standards Institute (AISI), Irvine, California, USA.

\section{References}

[1] Galindo-Rosales, F.J. (2016). Complex fluids in energy dissipating systems. Appl. Sci., 6(8), 206. https://doi.org/10.3390/app6080206

[2] Tian, T., \& Nakano, M. (2017). Design and testing of a rotational brake with shear thickening fluids. Smart Mater. Struct., 26(3), 035038. https://doi.org/10.1088/1361-665X/aa5a2c

[3] Cohen, D. (2008). Shear-Thickening Fluid Reinforced Fabrics for Use with an Expandable Spacecraft, Patent No. US20080296435A1.

[4] Decker, M.J., Halbach, C.J., Nam, C.H., Wagner, N.J., \& Wetzel, E.D. (2007). Stab resistance of shear thickening fluid (Stf)-treated fabrics. Compos. Sci. Technol., 67(3-4), 565-578. https://doi.org/10.1016/j.compscitech.2006.08.007

[5] Lee, Y.S., Wetzel, E.D., \& Wagner, N.J. (2003). The ballistic impact characteristics of Kevlar ${ }^{\circledR}$ woven fabrics impregnated with a colloidal shear thickening fluid. J. Mater. Sci., 38(13), 2825-2833. https://doi.org/10.1023/A:1024424200221

[6] Mewis, J., \& Wagner, N.J. (2012). Colloidal Suspension Rheology, Cambridge University Press.

[7] Wagner, N.J., \& Brady, J.F. (2009). Shear thickening in colloidal dispersions. Phys. Today, 62(10), 27-32. https://doi.org/10.1063/1.3248476

[8] Brown, E., \& Jaeger, H. M. (2014). Shear thickening in concentrated suspensions: phenomenology, mechanisms and relations to jamming. Rep. Prog. Phys., 77(4), 046602. https://doi.org/10.1088/0034-4885/77/4/046602

[9] Maranzano, B.J., \& Wagner, N.J. (2001). The effects of particle-size on reversible shear thickening of concentrated colloidal dispersions. J. Chem. Phys., 114(23), 10514-10527. https://doi.org/10.1063/1.1373687 
[10] Bender, J.W., \& Wagner, N.J. (1995). Optical measurement of the contributions of colloidal forces to the rheology of concentrated suspensions. J. Colloid Interface Sci., 172 (1), 171-184. https://doi.org/10.1006/jcis.1995.1240

[11] Bender, J., \& Wagner, N.J. (1996). Reversible shear thickening in monodisperse and bidisperse colloidal dispersions. J. Rheol., 40(5), 899-916.

https://doi.org/10.1122/1.550767

[12] Foss, D.R., \& Brady, J.F. (2000). Structure, diffusion and rheology of Brownian suspensions by Stokesian dynamics simulation. J. Fluid Mech., 407, 167-200. https://doi.org/10.1017/S0022112099007557

[13] Seto, R., Mari, R., Morris, J.F., \& Denn, M.M. (2013). Discontinuous shear thickening of frictional hard-sphere suspensions. Phys. Rev. Lett., 111(21), 218301. https://doi.org/10.1103/PhysRevLett.111.218301

[14] Guy, B., Hermes, M., \& Poon, W. (2015). Towards a unified description of the rheology of hard-particle suspensions. Phys. Rev. Lett., 115(8), 088304. https://doi.org/10.1103/PhysRevLett.115.088304

[15] Mari, R., Seto, R., Morris, J.F., \& Denn, M.M. (2014). Shear thickening, frictionless and frictional rheologies in non-Brownian suspensions. J. Rheol., 58(6), 1693-1724. https://doi.org/10.1122/1.4890747

[16] Clavaud, C., Bérut, A., Metzger, B., \& Forterre, Y. (2017). Revealing the frictional transition in shear-thickening suspensions. Proc. Natl. Acad. Sci. U.S.A., 114, 5147. https://doi.org/10.1073/pnas.1703926114

[17] Royer, J.R., Blair, D.L., \& Hudson, S.D. (2016). Rheological signature of frictional interactions in shear thickening suspensions. Phys. Rev. Lett., 116(18), 188301. https://doi.org/10.1103/PhysRevLett.116.188301

[18] Comtet, J., Chatté, G., Niguès, A., Bocquet, L., Siria, A., \& Colin, A. (2017). Pairwise frictional profile between particles determines discontinuous shear thickening transition in non-colloidal suspensions. Nat. Commun., 8, 15633.

https://doi.org/10.1038/ncomms15633

[19] Lin, N. Y., Guy, B. M., Hermes, M., Ness, C., Sun, J., Poon, W. C., \& Cohen, I. (2015). Hydrodynamic and contact contributions to continuous shear thickening in colloidal suspensions. Phys. Rev. Lett., 115(22), 228304. https://doi.org/10.1103/PhysRevLett.115.228304

[20] Wyart, M., \& Cates, M.E. (2014). Discontinuous shear thickening without inertia in dense non-Brownian suspensions. Phys. Rev. Lett., 112(9), 1.

https://doi.org/10.1103/PhysRevLett.112.098302 
[21] Cates, M.E., Wittmer, J.P., Bouchaud, J.P., \& Claudin, P. (1998). Jamming, force chains, and fragile matter. Phys. Rev. Lett., 81(9), 1841-1844.

https://doi.org/10.1103/PhysRevLett.81.1841

[22] Peters, I.R., Majumdar, S., \& Jaeger, H.M. (2016). Direct observation of dynamic shear jamming in dense suspensions. Nature, 532(7598), 214-217.

https://doi.org/10.1038/nature17167

[23] Waitukaitis, S.R., \& Jaeger, H.M. (2012). Impact-activated solidification of dense suspensions via dynamic jamming fronts. Nature, 487(7406), 205-209.

https://doi.org/10.1038/nature11187

[24] Han, E., Peters, I.R., \& Jaeger, H.M. (2016). High-speed ultrasound imaging in dense suspensions reveals impact-activated solidification due to dynamic shear jamming. Nat. Commun., 7, 12243. https://doi.org/10.1038/ncomms12243

[25] Maranzano, B.J., \& Wagner, N.J. (2001). The effects of interparticle interactions and particle size on reversible shear thickening: hard-sphere colloidal dispersions. J. Rheol., 45(5), 1205-1222. https://doi.org/10.1122/1.1392295

[26] Egres, R.G., Nettesheim, F., Wagner, N.J. (2006). Rheo-Sans investigation of acicularprecipitated calcium carbonate colloidal suspensions through the shear thickening transition. J. Rheol., 50(5), 685-709. https://doi.org/10.1122/1.2213245

[27] Egres, R.G., Wagner, N.J. (2005). The rheology and microstructure of acicular precipitated calcium carbonate colloidal suspensions through the shear thickening transition. J. Rheol., 49(3), 719-746. https://doi.org/10.1122/1.1895800

[28] Brown, E., Zhang, H., Forman, N.A., Maynor, B.W., Betts, D.E., DeSimone, J.M., \& Jaeger, H.M. (2011). Shear thickening and jamming in densely packed suspensions of different particle shapes. Phys. Rev. E, 84(3), 031408.

https://doi.org/10.1103/PhysRevE.84.031408

[29] Raghavan, S.R., Walls, H., \& Khan, S. A. (2000). Rheology of silica dispersions in organic liquids: new evidence for solvation forces dictated by hydrogen bonding. Langmuir, 16(21), 7920-7930. https://doi.org/10.1021/la991548q

[30] Gálvez, L.O., de Beer, S., van der Meer, D., \& Pons, A. (2017). Dramatic effect of fluid chemistry on cornstarch suspensions: linking particle interactions to macroscopic rheology. Phys. Rev. E: Stat. Phys., Plasmas, Fluids, Relat. Interdiscip. Top., 95(3), 030602. https://doi.org/10.1103/PhysRevE.95.030602

[31] Chu, B., Brady, A.T., Mannhalter, B.D., \& Salem, D.R. (2014). Effect of silica particle surface chemistry on the shear thickening behaviour of concentrated colloidal suspensions. J. Phys. D: Appl. Phys., 47(33), 335302.

https://doi.org/10.1088/0022-3727/47/33/335302 
[32] Shan, L., Tian, Y., Jiang, J., Zhang, X., \& Meng, Y. (2015). Effects of Ph on shear thinning and thickening behaviors of fumed silica suspensions. Colloids Surf., A, 464, 17. https://doi.org/10.1016/j.colsurfa.2014.09.040

[33] Krishnamurthy, L.-N., Wagner, N.J., \& Mewis, J. (2005). Shear thickening in polymer stabilized colloidal dispersions. J. Rheol., 49(6), 1347-1360.

https://doi.org/10.1122/1.2039867

[34] Shenoy, S.S., \& Wagner, N.J. (2005). Influence of medium viscosity and adsorbed polymer on the reversible shear thickening transition in concentrated colloidal dispersions. Rheol. Acta, 44(4), 360-371. https://doi.org/10.1007/s00397-004-0418-z

[35] Brown, E., Forman, N.A., Orellana, C.S., Zhang, H., Maynor, B.W., Betts, D.E., DeSimone, J.M., \& Jaeger, H.M. (2010). Generality of shear thickening in dense suspensions. Nat. Mater., 9(3), 220-224. https://doi.org/10.1038/nmat2627

[36] Gopalakrishnan, V., \& Zukoski, C. (2004). Effect of attractions on shear thickening in dense suspensions. J. Rheol., 48(6), 1321-1344. https://doi.org/10.1122/1.1784785

[37] Xu, K. (2004). Nonaqueous liquid electrolytes for lithium-based rechargeable batteries. Chem. Rev., 104(10), 4303-4418. https://doi.org/10.1021/cr030203g

[38] Ding, J., Tian, T., Meng, Q., Guo, Z., Li, W., Zhang, P., Ciacchi, F.T., Huang, J., \& Yang, W. (2013). Smart multifunctional fluids for lithium ion batteries: enhanced rate performance and intrinsic mechanical protection. Sci. Rep., 3, 2485.

https://doi.org/10.1038/srep02485

[39] Veith, G.M., Armstrong, B.L., Wang, H., Kalnaus, S., Tenhaeff, W.E., \& Patterson, M.L. (2017). Shear thickening electrolytes for high impact resistant batteries. ACS Energy Lett., 2(9), 2084-2088. https://doi.org/10.1021/acsenergylett.7b00511

[40] Shen, B.H., Veith, G.M., Armstrong, B.L., Tenhaeff, W.E., \& Sacci, R.L. (2017). Predictive design of shear-thickening electrolytes for safety considerations. $J$. Electrochem. Soc., 164(12), A2547-A2551. https://doi.org/10.1149/2.1171712jes

[41] Shen, B., Armstrong, B.L., Doucet, M., Heroux, L., Browning, J.F., Agamalian, M., Tenhaeff, W.E., \& Veith, G.M. (2018). Shear thickening electrolyte built from sterically stabilized colloidal particles. ACS Appl. Mater. Interfaces, 10(11), 9424-9434. https://doi.org/10.1021/acsami.7b19441

[42] Murphy, R.P., Hong, K.L., \& Wagner, N.J. (2016). Thermoreversible gels composed of colloidal silica rods with short range attractions. Langmuir, 32(33), 8424-8435. https://doi.org/10.1021/acs.langmuir.6b02107

[43] Belyakova, L.A., Varvarin, A.M., Lyashenko, D.Y., \& Roik, N.V. (2003). Study of 
interaction of Poly(1-Vinyl-2-Pyrrolidone) with a surface of highly dispersed amorphous silica. J. Colloid Interface Sci., 264(1), 2-6. https://doi.org/10.1016/S0021-9797(03)00395-3

[44] Ewoldt, R.H., Johnston, M.T., \& Caretta, L.M. (2015). Experimental challenges of shear rheology: how to avoid bad data. In Complex Fluids in Biological Systems, Springer, pp. 207-241.

[45] Mewis, J., \& Wagner, N.J. (2009). Thixotropy. Adv. Colloid Interface Sci., 147, 214-227. https://doi.org/10.1016/j.cis.2008.09.005

[46] Pednekar, S., Chun, J., \& Morris, J.F. (2017). Simulation of shear thickening in attractive colloidal suspensions. Soft Matter, 13(9), 1773-1779. https://doi.org/10.1039/C6SM02553F

[47] Zaccarelli, E., \& Poon, W.C. (2009). Colloidal glasses and gels: the interplay of bonding and caging. Proc. Natl. Acad. Sci. U.S.A., 106(36), 15203-15208.

https://doi.org/10.1073/pnas.0902294106

[48] Faroughi, S.A., \& Huber, C. (2015). A generalized equation for rheology of emulsions and suspensions of deformable particles subjected to simple shear at low Reynolds number. Rheol. Acta, 54(2), 85-108. https://doi.org/10.1007/s00397-014-0825-8

[49] Cwalina, C.D., Harrison, K.J., \& Wagner, N.J. (2016). Rheology of cubic particles suspended in a Newtonian fluid. Soft Matter, 12(20), 4654-4665.

https://doi.org/10.1039/C6SM00205F

[50] Trappe, V., Prasad, V., Cipelletti, L., Segre, P., \& Weitz, D. (2001). Jamming phase diagram for attractive particles. Nature, 411(6839), 772.

https://doi.org/10.1038/35081021

[51] Philipse, A. P. (1996). The random contact equation and its implications for (colloidal) rods in packings, suspensions, and anisotropic powders. Langmuir, 12(5), 1127-1133. https://doi.org/10.1021/la950671o

[52] Krishnamurthy, L.N., \& Wagner, N.J. (2005). Letter to the Editor: Comment on "Effect of attractions on shear thickening in dense suspensions". J. Rheol., 49(3), 799-803. https://doi.org/10.1122/1.1895797

[53] Krishnamurthy, L.-N., \& Wagner, N.J. (2005). The influence of weak attractive forces on the microstructure and rheology of colloidal dispersions. J. Rheol., 49(2), 475-499. https://doi.org/10.1122/1.1859792

[54] Eberle, A.P.R., Castaneda-Priego, R., Kim, J.M., \& Wagner, N.J. (2012). Dynamical arrest, percolation, gelation, and glass formation in model nanoparticle dispersions with 
thermoreversible adhesive interactions. Langmuir, 28(3), 1866-1878.

https://doi.org/10.1021/la2035054

[55] Gao, J., Ndong, R.S., Shiflett, M.B., Wagner, N.J. (2015). Creating nanoparticle stability in ionic liquid [C4mim][Bf4] by inducing solvation layering. ACS Nano, 9(3), 32433253. https://doi.org/10.1021/acsnano.5b00354

[56] Xu, K. (2004). Nonaqueous liquid electrolytes for lithium-based rechargeable batteries. Chem. Rev., 104(10), 4303-4417. https://doi.org/10.1021/cr030203g

[57] Yoon, I.-N., Song, H.-K., Won, J., Kang, \& Y.S. (2014). Shape dependence of $\mathrm{SiO}_{2}$ nanomaterials in a quasi-solid electrolyte for application in dye-sensitized solar cells. $J$. Phys. Chem. C, 118(8), 3918-3924. https://doi.org/10.1021/jp4104454

[58] Kuijk, A., van Blaaderen, A., \& Imhof, A. (2011). Synthesis of monodisperse, rodlike silica colloids with tunable aspect ratio. J. Am. Chem. Soc., 133(8), 2346-2349. https://doi.org/10.1021/ja109524h

[59] Lai, C.Y., Trewyn, B.G., Jeftinija, D.M., Jeftinija, K., Xu, S., Jeftinija, S., \& Lin, V.S.Y. (2003). A mesoporous silica nanosphere-based carrier system with chemically removable CdS nanoparticle caps for stimuli-responsive controlled release of neurotransmitters and drug molecules. J. Am. Chem. Soc., 125, 4451-4459. https://doi.org/10.1021/ja0286501

[60] Sinha, A., Chakraborty, A., \& Jana, N.R. (2014). Dextran-gated, multifunctional mesoporous nanoparticle for glucose-responsive and targeted drug delivery. ACS Appl. Mater. Interfaces, 6, 22183-22191. https://doi.org/10.1021/am505848p

[61] Kurtoglu, Y.E., Navath, R.S., Wang, B., Kannan, S., Romero, R., \& Kannan, R.M. (2009). Poly(amidoamine) dendrimer-drug Conjugates with disulfide linkages for intracellular drug delivery. Biomaterials, 30, 2112-2121.

https://doi.org/10.1016/j.biomaterials.2008.12.054

[62] Kesharwani, P., Jain, K., \& Jain, N. K. (2014). Dendrimer as nanocarrier for drug delivery. Prog. Polym. Sci., 39, 268-307.

https://doi.org/10.1016/j.progpolymsci.2013.07.005

[63] Müller, R. H., Mäder, K., \& Gohla, S. (2000). Solid lipid nanoparticles (SLN) for controlled drug delivery-a review of the state of the art. Eur. J. Pharm. Biopharm., 50, 161-177. https://doi.org/10.1016/S0939-6411(00)00087-4

[64] Muchow, M., Maincent, P., \& Müller, R.H. (2008). Lipid nanoparticles with a solid matrix (SLN, NLC, LDC) for oral drug delivery. Drug Dev. Ind. Pharm., 34, 1394-1405. https://doi.org/10.1080/03639040802130061

[65] Bae, Y., Fukushima, S., Harada, A., \& Kataoka, K. (2003). Design of environment- 
sensitive supramolecular assemblies for intracellular drug delivery: polymeric micelles that are responsive to intracellular $\mathrm{pH}$ change. Angew. Chem., Int. Ed., 42, 4640-4643. https://doi.org/10.1002/anie.200250653

[66] Nasongkla, N., Bey, E., Ren, J., Ai, H., Khemtong, C., Guthi, J.S., Chin, S.F., Sherry, A.D., Boothman, D.A., \& Gao, J. (2006). Multifunctional polymeric micelles as cancerTargeted, MRI-ultrasensitive drug delivery systems. Nano Lett., 6, 2427-2430. https://doi.org/10.1021/nl061412u

[67] Lian, T., \& Ho, R.J.Y. (2001). Trends and developments in liposome drug delivery systems. J. Pharm. Sci., 90, 667-680. https://doi.org/10.1002/jps.1023

[68] Allen, T.M., \& Cullis, P.R. (2013). Liposomal drug delivery systems: from concept to clinical applications. Adv. Drug Delivery Rev., 65, 36-48.

https://doi.org/10.1016/j.addr.2012.09.037

[69] Hamidi, M., Azadi, A., \& Rafiei, P. (2008). Hydrogel nanoparticles in drug delivery. Adv. Drug Delivery Rev., 60, 1638-1649. https://doi.org/10.1016/j.addr.2008.08.002

[70] Merino, S., Martin, C., Kostarelos, K., Prato, M., \& Vazquez, E. (2015). Nanocomposite hydrogels: 3D polymer-nanoparticle synergies for on-demand drug delivery. ACS Nano, 9, 4686-4697. https://doi.org/10.1021/acsnano.5b01433

[71] Chilkoti, A., Dreher, M.R., Meyer, D.E., \& Raucher, D. (2002). Targeted drug delivery by thermally responsive polymers. Adv. Drug Delivery Rev., 54, 613-630. https://doi.org/10.1016/S0169-409X(02)00041-8

[72] Sawant, R.M., Hurley, J.P., Salmaso, S., Kale, A., Tolcheva, E., Levchenko, T.S., \& Torchilin, V.P. (2006). "SMART" drug delivery systems: double-targeted pH-responsive pharmaceutical nanocarriers. Bioconjugate Chem., 17, 943-949. https://doi.org/10.1021/bc060080h

[73] Luo, Z., Cai, K., Hu, Y., Zhao, L., Liu, P., Duan, L., \& Yang, W. (2011). Mesoporous silica nanoparticles end-capped with collagen: redox-responsive nanoreservoirs for targeted drug delivery. Angew. Chem., Int. Ed., 50, 640-643.

https://doi.org/10.1002/anie.201005061

[74] Cheng, R., Feng, F., Meng, F., Deng, C., Feijen, J., \& Zhong, Z. (2011). Glutathioneresponsive nano-vehicles as a promising platform for targeted intracellular drug and gene delivery. J. Controlled Release, 152, 2-12. https://doi.org/10.1016/j.jconrel.2011.01.030

[75] Wang, Y., Wei, G., Zhang, X., Xu, F., Xiong, X., \& Zhou, S. (2017). A step-by-step multiple stimuli-responsive nanoplatform for enhancing combined chemo-photodynamic therapy. Adv. Mater., 29, 1605357. https://doi.org/10.1002/adma.201605357 
[76] Ulbrich, K., Hola, K., Subr, V., Bakandritsos, A., Tucek, J., \& Zboril, R. (2016). Targeted drug delivery with polymers and magnetic nanoparticles: covalent and noncovalent approaches, release control, and clinical studies. Chem. Rev., 116, 53385431. https://doi.org/10.1021/acs.chemrev.5b00589

[77] Moradi, E., Vllasaliu, D., Garnett, M., Falcone, F., \& Stolnik, S. (2012). Ligand density and clustering effects on endocytosis of folate modified nanoparticles. RSC Adv., 2, 30253033. https://doi.org/10.1039/c2ra01168a

[78] Saha, A., Basiruddin, S.K., Maity, A.R., \& Jana, N.R. (2013). Synthesis of nanobioconjugates with a controlled average number of biomolecules between 1 and 100 per nanoparticle and observation of multivalency dependent interaction with proteins and cells. Langmuir, 29, 13917-13924. https://doi.org/10.1021/la402699a

[79] Tang, Z., Li, D., Sun, H., Guo, X., Chen, Y., \& Zhou, S. (2014). Quantitative control of active targeting of nanocarriers to tumor cells through optimization of folate ligand density. Biomaterials, 35, 8015-8027. https://doi.org/10.1016/j.biomaterials.2014.05.091

[80] Dalal, C., Saha, A., \& Jana, N. R. (2016). Nanoparticle multivalency directed shifting of cellular uptake mechanism. J. Phys. Chem. C, 120, 6778-6786.

https://doi.org/10.1021/acs.jpcc.5b11059

[81] Dalal, C., \& Jana, N. R. (2017). Multivalency effect of TAT-peptide-functionalized nanoparticle in cellular endocytosis and subcellular trafficking. J. Phys. Chem. B, 121, 2942-2951. https://doi.org/10.1021/acs.jpcb.6b12182

[82] Schmaljohann, D. (2006). Thermo- and pH-responsive polymers in drug delivery. $A d v$. Drug Delivery Rev., 58, 1655-1670. https://doi.org/10.1016/j.addr.2006.09.020

[83] Gao, W., Chan, J.M., \& Farokhzad, O.C. (2010). pH-responsive nanoparticles for drug delivery. Mol. Pharmaceutics, 7, 1913-1920. https://doi.org/10.1021/mp100253e

[84] Saha, A., Mohanta, S.C., Deka, K., Deb, P., \& Devi, P.S. (2017). Surface-engineered multifunctional Eu: $\mathrm{Gd}_{2} \mathrm{O}_{3}$ nanoplates for targeted and $\mathrm{pH}$-responsive drug delivery and imaging applications. ACS Appl. Mater. Interfaces, 9, 4126-4141.

https://doi.org/10.1021/acsami.6b12804

[85] Zhang, L., Guo, R., Yang, M., Jiang, X., \& Liu, B. (2007). Thermo and pH dualresponsive nanoparticles for anti-cancer drug delivery. Adv. Mater., 19, 2988-2992. https://doi.org/10.1002/adma.200601817

[86] Bikram, M., \& West, J.L. (2008). Thermo-responsive systems for controlled drug delivery. Expert Opin. Drug Delivery, 5, 1077-1091.

https://doi.org/10.1517/17425247.5.10.1077 
[87] Liu, J., Detrembleur, C., Debuigne, A., De Pauw-Gillet, M.C., Mornet, S., Vander Elst, L., Laurent, S., Duguet, E., \& Jerome, C. (2014). Glucose-, pH- and thermo-responsive nanogels crosslinked by functional superparamagnetic maghemite nanoparticles as innovative drug delivery systems. J. Mater. Chem. B, 2, 1009-1023.

https://doi.org/10.1039/c3tb21272f

[88] Luo, Z., Cai, K., Hu, Y., Li, J., Ding, X., Zhang, B., Xu, D., Yang, W., \& Liu, P. (2012). Redox-responsive molecular nanoreservoirs for controlled intracellular anticancer drug delivery based on magnetic nanoparticles. Adv. Mater., 24, 431-435.

https://doi.org/10.1002/adma.201103458

[89] Wen, H., Dong, C., Dong, H., Shen, A., Xia, W., Cai, X., Song, Y., Li, X., Li, Y., \& Shi, D. (2012). Engineered redox-responsive PEG detachment mechanism in PEGylated nano-graphene oxide for intracellular drug delivery. Small, 8, 760-769.

https://doi.org/10.1002/smll.201101613

[90] Shao, Y., Shi, C., Xu, G., Guo, D., \& Luo, J. (2014). Photo and redox dual responsive reversibly cross-linked nanocarrier for efficient tumor-targeted drug delivery. ACS Appl. Mater. Interfaces, 6, 10381-10392. https://doi.org/10.1021/am501913m

[91] Shi, C., Guo, X., Qu, Q., Tang, Z., Wang, Y., \& Zhou, S. (2014). Actively targeted delivery of anticancer drug to tumor cells by redox-responsive star-shaped micelles. Biomaterials, 35, 8711-8722. https://doi.org/10.1016/j.biomaterials.2014.06.036

[92] Yui, N., Okano, T., \& Sakurai, Y. (1993). Photo-responsive degradation of heterogeneous hydrogels comprising crosslinked hyaluronic acid and lipid microspheres for temporal drug delivery. J. Controlled Release, 26, 141-145.

https://doi.org/10.1016/0168-3659(93)90113-J

[93] Mathiyazhakan, M., Wiraja, C., \& Xu, C. (2018). A concise review of gold nanoparticlesbased photo-responsive liposomes for controlled drug delivery. Nano-Micro Lett., 10, 10. https://doi.org/10.1007/s40820-017-0166-0

[94] Yang, Y., Aw, J., Chen, K., Liu, F., Padmanabhan, P., Hou, Y., Cheng, Z., \& Xing, B. (2011). Enzyme-responsive multifunctional magnetic nanoparticles for tumor intracellular drug delivery and imaging. Chem. - Asian J., 6, 1381-1389.

https://doi.org/10.1002/asia.201000905

[95] Hu, Q., Katti, P.S., \& Gu, Z. (2014). Enzyme-responsive nanomaterials for controlled drug delivery. Nanoscale, 6, 12273-12286. https://doi.org/10.1039/C4NR04249B

[96] Huang, J., Shu, Q., Wang, L., Wu, H., Wang, A.Y., \& Mao, H. (2015). Layer-by-layer assembled milk protein coated magnetic nanoparticle enabled oral drug delivery with high stability in stomach and enzyme-responsive release in small intestine. Biomaterials, 39, 105-113. https://doi.org/10.1016/j.biomaterials.2014.10.059 
[97] Kim, J., Kim, H.S., Lee, N., Kim, T., Kim, H., Yu, T., Song, I.C., Moon, W.K., \& Hyeon, T. (2008). Multifunctional uniform nanoparticles composed of a magnetite nanocrystal core and a mesoporous silica shell for magnetic resonance and fluorescence imaging and for drug delivery. Angew. Chem., Int. Ed., 47, 8438-8441.

https://doi.org/10.1002/anie.200802469

[98] Liong, M., Lu, J., Kovochich, M., Xia, T., Ruehm, S.G., Nel, A.E., Tamanoi, F., \& Zink, J.I. (2008). Multifunctional inorganic nanoparticles for imaging, targeting, and drug delivery. ACS Nano, 2, 889-896. https://doi.org/10.1021/nn800072t

[99] Taylor-Pashow, K.M.L., Della Rocca, J., Xie, Z., Tran, S., \& Lin, W. (2009). Postsynthetic modifications of iron-carboxylate nanoscale metal-organic frameworks for imaging and drug delivery. J. Am. Chem. Soc., 131, 14261-14263.

https://doi.org/10.1021/ja906198y

[100] Maity, A.R., Saha, A., Roy, A., \& Jana, N. R. (2013). Folic acid functionalized nanoprobes for fluorescence-, dark-field-, and dual-imaging-based selective detection of cancer cells and tissue. ChemPlusChem, 78, 259-267.

https://doi.org/10.1002/cplu.201200296

[101] Sun, C., Lee, J.S.H., \& Zhang, M. (2008). Magnetic nanoparticles in MR imaging and drug delivery. Adv. Drug Delivery Rev., 60, 1252-1265.

https://doi.org/10.1016/j.addr.2008.03.018

[102] Yang, X., Hong, H., Grailer, J.J., Rowland, I.J., Javadi, A., Hurley, S.A., Xiao, Y., Yang, Y., Zhang, Y., Nickles, R.J., Cai, W., Steeber, D.A., \& Gong, S. (2011). cRGDfunctionalized, DOX-conjugated, and 64Cu-labeled superparamagnetic iron oxide nanoparticles for targeted anticancer drug delivery and PET/MR imaging. Biomaterials, 32, 4151-4160. https://doi.org/10.1016/j.biomaterials.2011.02.006

[103] Mukai, H., Ozaki, D., Cui, Y., Kuboyama, T., Yamato-Nagata, H., Onoe, K., Takahashi, M., Wada, Y., Imanishi, T., Kodama, T., Obika, S., Suzuki, M., Doi, H., \& Watanabe, Y. (2014). Quantitative evaluation of the improvement in the pharmacokinetics of a nucleic acid drug delivery system by dynamic PET imaging with 18F-incorporated oligodeoxynucleotides. J. Controlled Release, 180, 92-99.

https://doi.org/10.1016/j.jconrel.2014.02.014

[104] Hendriks, B., Shields, A., Siegel, B.A., Miller, K., Munster, P., Ma, C., Campbell, K., Moyo, V., Wickham, T., \& Lo Russo, P. (2014). PET/CT imaging of $64 \mathrm{Cu}$-labelled HER2 liposomal doxorubicin (64Cu-MM-302) quantifies variability of liposomal drug delivery to diverse tumor lesions in HER2-positive breast cancer patients. Ann. Oncol., 25, i19. https://doi.org/10.1093/annonc/mdu068.1 
[105] Lin, W., Yao, N., Zhang, X., \& Zhang, L. (2017). pH-sensitive polymer-gold nanohybrid system for antitumor drug delivery and CT imaging. J. Controlled Release, 259, e14110. https://doi.org/1016/j.jconrel.2017.03.286

[106] Bridot, J.L., Faure, A.C., Laurent, S., Riviere, C., Billotey, C., Hiba, B., Janier, M., Josserand, V., Coll, J.L., Vander Elst, L., Muller, R., Roux, S., Perriat, P., \& Tillement, O. (2007). Hybrid gadolinium oxide nanoparticles: multimodal contrast agents for in vivo imaging. J. Am. Chem. Soc., 129, 5076-5084. https://doi.org/10.1021/ja068356j

[107] Kim, J., Piao, Y., \& Hyeon, T. (2009). Multifunctional nanostructured materials for multimodal imaging, and simultaneous imaging and therapy. Chem. Soc. Rev., 38, 372390. https://doi.org/10.1039/B709883A

[108] Saha, A., Basiruddin, S.K., Sarkar, R., Pradhan, N., \& Jana, N.R. (2009). Functionalized plasmonic-fluorescent nanoparticles for imaging and detection. J. Phys. Chem. C, 113, 18492-18498. https://doi.org/10.1021/jp904791h

[109] Qi, J., Han, M.S., Chang, Y.C., \& Tung, C.H. (2011). Developing visible fluorogenic 'click-on' dyes for cellular imaging. Bioconjugate Chem., 22, 1758-1762. https://doi.org/10.1021/bc200282t

[110] Zhu, S., Yang, Q., Antaris, A.L., Yue, J., Ma, Z., Wang, H., Huang, W., Wan, H., Wang, J., Diao, S., Zhang, B., Li, X., Zhong, Y., Yu, K., Hong, G., Luo, J., Liang, Y., \& Dai, H. (2017). Molecular imaging of biological systems with a clickable dye in the broad 800- to 1,700-nm near-infrared window. Proc. Natl. Acad. Sci. U.S.A., 114, 962-967. https://doi.org/10.1073/pnas.1617990114

[111] Bruchez, M., Jr., Moronne, M., Gin, P., Weiss, S., \& Alivisatos, A.P. (1998). Semiconductor nanocrystals as fluorescent biological labels. Science, 281, 2013-2016. https://doi.org/10.1126/science.281.5385.2013

[112] Kim, S., Lim, Y.T., Soltesz, E.G., De Grand, A.M., Lee, J., Nakayama, A., Parker, J.A., Mihaljevic, T., Laurence, R.G., Dor, D.M., Cohn, L.H., Bawendi, M.G., \& Frangioni, J.V. (2004). Near-infrared fluorescent type II quantum dots for sentinel lymph node mapping. Nat. Biotechnol., 22, 93-97. https://doi.org/10.1038/nbt920

[113] Derfus, A.M., Chan, W.C.W., \& Bhatia, S.N. (2004). Intracellular delivery of quantum dots for live cell labeling and organelle tracking. Adv. Mater., 16, 961-966. https://doi.org/10.1002/adma.200306111

[114] Gnach, A., \& Bednarkiewicz, A. (2012). Lanthanide-doped up-converting nanoparticles: merits and challenges. Nano Today, 7, 532-563. https://doi.org/10.1016/j.nantod.2012.10.006

[115] Park, Y., Kim, H.M., Kim, J.H., Moon, K.C., Yoo, B., Lee, K.T., Lee, N., Choi, Y., Park, 
W., Ling, D., Na, K., Moon, W.K., Choi, S.H., Park, H.S., Yoon, S.Y., Suh, Y.D., Lee, S.H., \& Hyeon, T. (2012). Theranostic probe based on lanthanide-doped nanoparticles for simultaneous in vivo dual-modal imaging and photodynamic therapy. Adv. Mater., 24, 5755-5761. https://doi.org/10.1002/adma.201202433

[116] Ray, S.C., Saha, A., Jana, N.R., \& Sarkar, R. (2009). Fluorescent carbon nanoparticles: synthesis, characterization, and bioimaging application. J. Phys. Chem. C, 113, 1854618551. https://doi.org/10.1021/jp905912n

[117] Bhunia, S.K., Saha, A., Maity, A.R., Ray, S.C., \& Jana, N.R. (2013). Carbon nanoparticle-based fluorescent bioimaging probes. Sci. Rep., 3, 1473. https://doi.org/10.1038/srep01473

[118] Das, P., Saha, A., Maity, A.R., Ray, S.C., Jana, N.R. (2013). Silicon nanoparticle based fluorescent biological label via low temperature thermal degradation of chloroalkylsilane. Nanoscale, 5, 5732-5737. https://doi.org/10.1039/c3nr00932g

[119] Pan, D., Zhang, J., Li, Z., \& Wu, M. (2010). Hydrothermal route for cutting graphene sheets into blue-luminescent graphene quantum dots. Adv. Mater., 22, 734-738. https://doi.org/10.1002/adma.200902825

[120] Shen, J., Zhu, Y., Yang, X., \& Li, C. (2012). Graphene quantum dots: emergent nanolights for bioimaging, sensors, catalysis and photovoltaic devices. Chem. Commun., 48, 3686-3699. https://doi.org/10.1039/c2cc00110a

[121] Renikuntla, B.R., Rose, H.C., Eldo, J., Waggoner, A. S., \& Armitage, B.A. (2004). Improved photostability and fluorescence properties through polyfluorination of a cyanine dye. Org. Lett., 6, 909-912. https://doi.org/10.1021/o1036081w

[122] Guo, L., \& Gai, F. (2013). Simple method to enhance the photostability of the fluorescence reporter R6G for prolonged single molecule studies. J. Phys. Chem. A, 117, 6164-6170. https://doi.org/10.1021/jp4003643

[123] Grimm, J.B., English, B.P., Chen, J., Slaughter, J.P., Zhang, Z., Revyakin, A., Patel, R., Macklin, J.J., Normanno, D., Singer, R.H., Lionnet, T., \& Lavis, L.D. (2015). A general method to improve fluorophores for live-cell and single-molecule microscopy. Nat. Methods, 12, 244-250. https://doi.org/10.1038/nmeth.3256

[124] Jiao, L., Song, F., Zhang, B., Ning, H., Cui, J., \& Peng, X. (2017). Improving the brightness and photostability of NIR fluorescent silica nanoparticles through rational fine-tuning of the covalent encapsulation methods. J. Mater. Chem. B, 5, 5278-5283, https://doi.org/10.1039/C7TB00856B

[125] Kim, J., Lee, J. E., Lee, J., Yu, J. H., Kim, B. C., An, K., Hwang, Y., Shin, C. H., Park, J. G., Kim, J., \& Hyeon, T. (2006). Magnetic fluorescent delivery vehicle using uniform 
mesoporous silica spheres embedded with monodisperse magnetic and semiconductor nanocrystals. J. Am. Chem. Soc., 128, 688-689. https://doi.org/10.1021/ja0565875

[126] Corr, S.A., Rakovich, Y.P., \& Gun'ko, Y.K. (2008). Multifunctional magneticfluorescent nanocomposites for biomedical applications. Nanoscale Res. Lett., 3, 87-104. https://doi.org/10.1007/s11671-008-9122-8

[127] Saha, A., Basiruddin, S. K., Pradhan, N., \& Jana, N. R. (2010). Ligand exchange approach in deriving magnetic-fluorescent and magnetic-plasmonic hybrid nanoparticle. Langmuir, 26, 4351-4356. https://doi.org/10.1021/la903428r

[128] Jin, Y., \& Gao, X. (2009). Plasmonic fluorescent quantum dots. Nat. Nanotechnol., 4, 571-576. https://doi.org/10.1038/nnano.2009.193

[129] Bigall, N.C., Parak, W.J., \& Dorfs, D. (2012). Fluorescent, magnetic and plasmonichybrid multifunctional colloidal nano objects. Nano Today, 7, 282-296.

https://doi.org/10.1016/j.nantod.2012.06.007

[130] Xu, Z., Hou, Y., \& Sun, S. (2007). Magnetic core/shell $\mathrm{Fe}_{3} \mathrm{O}_{4} / \mathrm{Au}$ and $\mathrm{Fe}_{3} \mathrm{O}_{4} / \mathrm{Au} / \mathrm{Ag}$ nanoparticles with tunable plasmonic properties. J. Am. Chem. Soc., 129, 8698-8699. https://doi.org/10.1021/ja073057v

[131] Fan, Z., Shelton, M., Singh, A. K., Senapati, D., Khan, S. A., \& Ray, P. C. (2012). Multifunctional plasmonic shell-magnetic core nanoparticles for targeted diagnostics, isolation, and photothermal destruction of tumor cells. ACS Nano, 6, 1065-1073. https://doi.org/10.1021/nn2045246

[132] Stankovich, S., Dikin, D.A., Dommett, G.H.B., Kohlhaas, K.M., Zimney, E.J., Stach, E.A., Piner, R.D., Nguyen, S.T., \& Ruoff, R.S. (2006). Graphene-based composite materials. Nature, 442, 282-286. https://doi.org/10.1038/nature04969

[133] Huang, X., Qi, X., Boey, F., \& Zhang, H. (2012). Graphene-based composites. Chem. Soc. Rev., 41, 666-686. https://doi.org/10.1039/C1CS15078B

[134] Jiang, J., Gu, H., Shao, H., Devlin, E., Papaefthymiou, G.C., \& Ying, J.Y. (2008). Bifunctional $\mathrm{Fe}_{3} \mathrm{O}_{4}$ - $\mathrm{Ag}$ heterodimer nanoparticles for two-photon fluorescence imaging and magnetic manipulation. Adv. Mater., 20, 4403-4407.

https://doi.org/10.1002/adma.200800498

[135] Deng, S., Ruan, G., Han, N., \& Winter, J.O. (2010). Interactions in fluorescent-magnetic heterodimer nanocomposites. Nanotechnology, 21, 145605.

https://doi.org/10.1088/0957-4484/21/14/145605

[136] Basiruddin, S.K., Saha, A., Pradhan, N., \& Jana, N.R. (2010). Advances in coating chemistry in deriving soluble functional nanoparticle. J. Phys. Chem. C, 114, 1100911017. https://doi.org/10.1021/jp100844d 
[137] Jia, G., You, H., Liu, K., Zheng, Y., Guo, N., \& Zhang, H. (2010). Highly uniform $\mathrm{Gd}_{2} \mathrm{O}_{3}$ hollow microspheres: template-directed synthesis and luminescence properties. Langmuir, 26, 5122-5128. https://doi.org/10.1021/la903584j

[138] Ahren, M., Selegard, L., Klasson, A., Soderlind, F., Abrikossova, N., Skoglund, C., Bengtsson, T., Engstrom, M., Kall, P.O., \& Uvdal, K. (2010). Synthesis and characterization of PEGylated $\mathrm{Gd}_{2} \mathrm{O}_{3}$ nanoparticles for MRI contrast enhancement. Langmuir, 26, 5753-5762. https://doi.org/10.1021/1a903566y

[139] Dosev, D., Nichkova, M., Liu, M., Guo, B., Liu, G.Y., Hammock, B.D., \& Kennedy, I.M. (2005). Application of luminescent $\mathrm{Eu}: \mathrm{Gd}_{2} \mathrm{O}_{3}$ nanoparticles to the visualization of protein micropatterns. J. Biomed. Opt., 10, 064006. https://doi.org/10.1117/1.2136347

[140] Paik, T., Gordon, T.R., Prantner, A.M., Yun, H., \& Murray, C.B. (2013). Designing tripodal and triangular gadolinium oxide nanoplates and self-assembled nanofibrils as potential multimodal bioimaging probes. ACS Nano, 7, 2850-2859.

https://doi.org/10.1021/nn4004583

[141] Zhou, C., Wu, H., Huang, C., Wang, M., \& Jia, N. (2014). Facile synthesis of singlephase mesoporous $\mathrm{Gd}_{2} \mathrm{O}_{3}:$ Eu nanorods and their application for drug delivery and multimodal imaging. Part. Part. Syst. Charact., 31, 675-684.

https://doi.org/10.1002/ppsc.201300342

[142] Kim, W.J., Gwag, J.S., Kang, J.G., \& Sohn, Y. (2014). Photoluminescence imaging of $\mathrm{Eu}(\mathrm{III}), \mathrm{Eu}(\mathrm{III}) / \mathrm{Ag}, \mathrm{Eu}(\mathrm{III}) / \mathrm{Tb}(\mathrm{III})$, and $\mathrm{Eu}(\mathrm{III}) / \mathrm{Tb}(\mathrm{III}) / \mathrm{Ag}$-doped $\mathrm{Gd}(\mathrm{OH})_{3}$ and $\mathrm{Gd}_{2} \mathrm{O}_{3}$ nanorods. Ceram. Int., 40, 12035-12044. https://doi.org/10.1016/j.ceramint.2014.04.043

[143] Wawrzynczyk, D., Samoc, M., \& Nyk, M. (2015). Controlled synthesis of luminescent $\mathrm{Gd}_{2} \mathrm{O}_{3}$ : $\mathrm{Eu}^{3+}$ nanoparticles by alkali ion doping. CrystEngComm, 17, 1997-2003. https://doi.org/10.1039/C4CE02500H

[144] Geng, Y., Dalhaimer, P., Cai, S., Tsai, R., Tewari, M., Minko, T., \& Discher, D.E. (2007). Shape effects of filaments versus spherical particles in flow and drug delivery. Nat. Nanotechnol., 2, 249-255. https://doi.org/10.1038/nnano.2007.70

[145] Toy, R., Peiris, P.M., Ghaghada, K.B., \& Karathanasis, E. (2014). Shaping cancer nanomedicine: the effect of particle shape on the in vivo journey of nanoparticles. Nanomedicine, 9, 121-134. https://doi.org/10.2217/nnm.13.191

[146] Godwin, A.K., Meister, A., O’dwyer, P.J., Huang, C.S., Hamilton, T.C., \& Anderson, M.E. (1992). High resistance to cisplatin in human ovarian cancer cell lines is associated with marked increase of glutathione synthesis. Proc. Natl. Acad. Sci. U.S.A., 89, 30703074. https://doi.org/10.1073/pnas.89.7.3070 
[147] Balendiran, G.K., Dabur, R., \& Fraser, D. (2004). The role of glutathione in cancer. Cell Biochem. Funct., 22, 343-352. https://doi.org/10.1002/cbf.1149

[148] Gronow, M. (2010). Studies on the non-protein thiols of a human prostatic cancer cell line: glutathione content. Cancers, 2, 1092-1106. https://doi.org/10.3390/cancers2021092

[149] Guo, X., Wei, X., Jing, Y., \& Zhou, S. (2015). Size changeable nanocarriers with nuclear targeting for effectively overcoming multidrug resistance in cancer therapy. Adv. Mater., 27, 6450-6456. https://doi.org/10.1002/adma.201502865

[150] Heidari, A., \& Brown, C. (2015). Study of composition and morphology of cadmium oxide (CdO) nanoparticles for eliminating cancer cells, J Nanomed Res., 2(5), 20 pp.

[151] Heidari, A. (2016). Pharmacogenomics and pharmacoproteomics studies of phosphodiesterase-5 (PDE5) inhibitors and paclitaxel albumin-stabilized nanoparticles as sandwiched anti-cancer nano drugs between two DNA/RNA molecules of human cancer cells, J Pharmacogenomics Pharmacoproteomics 7, e153.

[152] Heidari, A., \& Brown, C. (2015). Study of surface morphological, phytochemical and structural characteristics of rhodium (III) oxide $\left(\mathrm{Rh}_{2} \mathrm{O}_{3}\right)$ nanoparticles, International Journal of Pharmacology, Phytochemistry and Ethnomedicine, 1(1), 15-19.

[153] Heidari, A. (2016). Extraction and preconcentration of N-tolyl-sulfonyl-phosphoramidsaeure-dichlorid as an anti-cancer drug from plants: a pharmacognosy study, $J$ Pharmacogn Nat Prod 2, e103.

[154] Heidari, A. (2016). A chemotherapeutic and biospectroscopic investigation of the interaction of double-standard DNA/RNA-binding molecules with cadmium oxide (CdO) and rhodium (III) oxide $\left(\mathrm{Rh}_{2} \mathrm{O}_{3}\right)$ nanoparticles as anti-cancer drugs for cancer cells' treatment, Chemo Open Access 5, e129.

[155] Heidari, A. (2016). Linear and non-linear quantitative structure-anti-cancer-activity relationship (QSACAR) study of hydrous ruthenium (IV) oxide $\left(\mathrm{RuO}_{2}\right)$ nanoparticles as non-nucleoside reverse transcriptase inhibitors (NNRTIs) and anti-cancer nano drugs, $J$ Integr Oncol 5, e110.

[156] Heidari, A. (2016). Genomics and proteomics studies of zolpidem, necopidem, alpidem, saripidem, miroprofen, zolimidine, olprinone and abafungin as anti-tumor, peptide antibiotics, antiviral and central nervous system (CNS) drugs, J Data Mining Genomics \& Proteomics 7, e125. 